remains low. Shared decision-making (SDM) may increase awareness and help patients select and follow through with informed options for diabetes prevention that are aligned with their preferences.The objective was to test the effectiveness of a prediabetes SDM intervention. METHODS/STUDY POPULATION: This was a cluster-randomized controlled trial in 20 primary care clinics within a large regional health system. Participants were overweight/ obese adults with prediabetes (BMI $>24 \mathrm{~kg} / \mathrm{m} 2$ and $\mathrm{HbA} 1 \mathrm{c} 5.7-6.4 \%$ ) were enrolled from 10 SDM intervention clinics. Propensity score matching was used to identify control patients from 10 usual care clinics.Intervention clinic patients were invited to participate in a face-to-face SDM visit with a pharmacist who used a decision aid (DA) to describe prediabetes and four possible options for diabetes prevention; DPP, DPP +/- metformin, metformin only, or usual care. RESULTS/ANTICIPATED RESULTS: Uptake of DPP and/or metformin was higher among SDM participants $(n=351)$ than controls receiving usual care $(\mathrm{n}=1,028 ; 38 \%$ vs. $2 \%, \mathrm{p}<.001)$. At 12-months follow-up, adjusted weight loss (lbs.) was greater among SDM participants than controls $(-5.3$ vs. $-0.2, \mathrm{p}<.001)$. DISCUSSION/SIGNIFICANCE OF IMPACT: A prediabetes SDM intervention led by pharmacists increased patient engagement in evidence-based options for diabetes prevention and was associated with significantly greater uptake of DPP and/or metformin at 4 -months and weight loss at 12 -months. Prediabetes SDM may be a promising approach to enhance prevention efforts among patients at increased risk.

3068

Effects of exercise and a very low fat diet in metabolically abnormal obese adults

George Schweitzer ${ }^{1}$, Monica Kearney, Gordon Smith and

Samuel Klein

${ }^{1}$ Washington University in St. Louis, Institute Of Clinical and

Translational Sciences

OBJECTIVES/SPECIFIC AIMS: People with metabolically abnormal obesity (MAO), defined as those with insulin resistance and high intrahepatic triglyceride, are at high risk for developing type 2 diabetes and cardiovascular disease. Weight loss through reduced energy intake and increased physical activity has profound impacts on improving cardiometabolic function. However, the specific additional effects of exercise training with diet-induced weight loss on metabolic function are equivocal. METHODS/STUDY POPULATION: A comparative trial is ongoing in MAO adults undergoing $8-10 \%$ weight loss induced by a very-low fat plantbased $(\mathrm{PB})$ diet with structured exercise training $(\mathrm{n}=8)$ compared to the same weight loss induced by the $\mathrm{PB}$ diet alone $(n=3)$. RESULTS/ANTICIPATED RESULTS: Preliminary results indicate that, $\mathrm{PB}$ diet with or without exercise training results in significant weight loss concomitant with enhanced insulin sensitivity, reduced intrahepatic triglyceride, reduced 24-hour postprandial glucose response, reduced fat mass, and reduced diastolic blood pressure. Those undergoing PB diet with exercise training had greater improvements in muscular strength and cardiorespiratory fitness than those undergoing PB diet alone. Differences between intervention groups for other cardiometabolic measures are not yet known. DISCUSSION/SIGNIFICANCE OF IMPACT: Each of the interventions resulted in improved cardiometabolic measures; however the extent of the differences between the interventions is not yet clear. It is hypothesized that compared with weight loss induced by a PB diet, the same weight loss induced by a PB diet and structured exercise training will i) cause greater improvement in skeletal muscle insulin sensitivity, ii) will attenuate the usual decline in muscle mass while increasing strength, and iii) result in greater increases in left ventricular diastolic function. The long-term objective of this proposal is to provide a foundation for future studies evaluating mechanisms for the effects of exercise in cardiometabolic disease prevention and therapy.

3447

\section{Effects of intranasal ketamine on uncontrolled cancer related pain}

Vinita Singh ${ }^{1}$ and Donald Harvey

${ }^{1}$ Emory University

OBJECTIVES/SPECIFIC AIMS: If intranasal ketamine can be utilized for pain control in cancer patients, this could provide them with superior analgesia and better quality of life, without the risk of significant respiratory depression associated with opioid medications. We seek to obtain preliminary data via a clinical trial addressing safety, feasibility, and utility of this novel technique for the treatment of persistent uncontrolled cancer pain. These findings would be an important initial step towards testing the effectiveness of intranasal ketamine as a non-opioid medication for cancer pain used as potential maintenance outpatient therapy. These initial findings would be applied to a subsequent trial to determine the effectiveness and associated toxicities of ketamine in a larger sample of cancer patients, and address the compelling need to identify new, successful management therapies for cancer pain. Specific Aims: 1. To evaluate (pharmacodynamic) effects of NAS ketamine on Patient Reported Outcomes (PROs), such as pain scores, side effects, depression, quality of life, and functional status. A clinical trial will be conducted where NAS ketamine will be given to a sample of patients with cancer related pain. Patient Reported Outcomes (PROs), such as pain scores, depression, quality of life, and functional status will be noted on Numerical Pain Rating Scale (NPRS), Montgomery Asberg Depression Rating Scale (MADRS), and Edmonton Symptom Assessment (ESAS), Eastern Cooperative Oncology Group (ECOG) and Patient Reported Outcome Measurement Information System (PROMIS) scales respectively. 1. To measure pharmacokinetics of NAS ketamine through analysis of ketamine and its metabolite norketamine to determine pharmacokinetic properties. During this clinical trial blood samples will be drawn at specified intervals and sent for analysis. 3. To determine opioid sparing effect of NAS ketamine. Opioid use will be measured by documenting use of rescue medications prior to and during the study and by evaluating total opioid consumption prior to and during the study. METHODS/STUDY POPULATION: Study sample: In the search for improved therapies for chronic cancer pain, medications with novel mechanisms of action have been sought. One such promising pharmacologic approach is ketamine. We specifically intend to measure utility of ketamine in patients with pain related to cancer or cancer treatment. Ketamine has shown to reverse central sensitization and opioid tolerance in rat models. Since ketamine is Scheduled III in United States and has abuse potential, we do not intend for ketamine to replace opioids, but use in patients who have failed opioid therapy. Since the investigators of the study practice at Emory, subjects will be from oncology and pain clinics (the supportive oncology clinic, oncology clinics, the pain clinic and Acute Pain Service) at Emory. The trial will be conducted at the Phase 1 Unit of the Winship Cancer Institute (WCI) at 
Emory. Subjects may be identified and contacted via telephone with information about the study prior to their next clinic appointment in order to allow time for them to consider the study. Eligibility criteria: Patients will be eligible to participate if they are: 1. Adults with uncontrolled cancer related pain a. Male and female subjects at least 18 years of age. b. Patients with uncontrolled pain related to cancer or cancer treatment. c. Uncontrolled pain will be defined as i. pain which persists for more than 7 days and is rated $>/=4$ on NPRS, and/or ii. use of breakthrough medication more than 4 times in 24 hours d. Failed other pain medications such non-steroidal antiinflammatories such as ibuprofen, acetaminophen, opioids such as tramadol, hydrocodone, oxycodone etc. and antineuropathics such as gabapentin. 2. Able to provide informed consent a. Patients who are able to understand written and verbal English. Patients will be excluded from the study if they have any of the following: 1. Conditions increasing the risk of side effects from ketamine a. Conditions not safe due to cardiovascular effects of ketamine i. Presence of severe cardiac disease-EF $<15 \%$ in patients with known history of cardiac disease ii. Uncontrolled Stage 2 hypertension or greater (systolic blood pressure $>160$ and/or diastolic blood pressure $>100$ ) iii. Baseline tachycardia, HR $>100 \mathrm{~b}$. Conditions not safe due to potential effect of ketamine on intracranial and intraocular pressure i. Presence of elevated ICP ii. Uncontrolled glaucoma c. Presence of uncontrolled depression or other psychiatric comorbidity with psychosis 2 . Conditions not safe due to potential side effects reported in ketamine abusers a. History of liver disease b. History of interstitial cystitis 3 . Conditions where delivery of intranasal medications may be unreliable a. Active allergic or infectious rhinitis b. Patients with lesions of nasal mucosa 4 . Conditions where fetus may be exposed to ketamine in utero (ketamine is category $\mathrm{C}$ medication) a. Pregnant women, nursing mothers and women of childbearing potential not using contraception known to be highly effective. b. Highly effective contraception methods include combination of any two of the following: Use of oral, injected or implanted hormonal methods of contraception or; Placement of an intrauterine device (IUD) or intrauterine system (IUS); Barrier methods of contraception: condom or occlusive cap (diaphragm or cervical/vault caps) with spermicidal foam/gel/film/cream/ vaginal suppository; Total abstinence; Male/female sterilization. 5. Conditions with medication abuse potential a. Illicit substance abuse within the past 6 months b. Documented history of medication abuse/misuse (e.g. Unsanctioned dose escalation, broken opioid agreement etc.) 6. Conditions where ketamine metabolism may be altered, resulting in erroneous dose response relationship a. Clinical requirement for medications that are concurrent inducers or strong inhibitors of CYP3A4. CYP3A4 substrates are allowed. (Ketamine is metabolized by CYP3A4) Study sample limitations: Subject factors that may affect the final resultant study sample of subjects with full data for analysis. 1. Subjects who may not get pain relief with ketamine may not follow up and resulting incomplete data not eligible for analysis that may erroneously enhance positive effect of ketamine on pain relief. To account for this effort will be made to document the reason for lack of follow-up by contacting patient via telephone or at next scheduled clinic visit within Emory Healthcare. 2. Since patients coming to Emory are typically insured, the study will not adequately capture indigent population. It is not the intention of the current study to investigate differences in pain characteristics or responses of patients with insurance vs indigent population and will need to be addressed via future trials. Since this is a single center trial, the results of this trial might lack external validity required to support widespread changes in practice. This will be a pilot trial to figure out likely most efficacious dose. If this trial is successful, a multi-site randomized clinical trial will be conducted next. Primary Study Measures Primary exposure Intranasal Ketamine for cancer related pain Ketamine is an FDA approved anesthetic with amnesic, analgesic, dissociative, and sedative properties. It is unique among anesthetic agents in that it does not depress cardiovascular and respiratory systems. Ketamine is a noncompetitive, antagonist of N-methyl$\mathrm{D}$-aspartate (NMDA) receptors that blocks the NMDA channel in the open state by binding to the phencyclidine (PCP) site located within the lumen of the channel. Antagonism of NMDA receptors produces antinociception of persistent or neuropathic pain in animal models and analgesia in pain states in humans. The NMDA receptor is believed to play a role in the development of opioid tolerance and ketamine has been shown in a rat model to prevent fentanyl-induced hyperalgesia and subsequent acute morphine tolerance 5. Ketamine also interacts at a number of other receptor sites to block pain. Some of these sites include voltage-sensitive calcium channels, depression of sodium channels, modulation of cholinergic neurotransmission, and inhibition of uptake of serotonin and norepinephrine. Ketamine also interacts with kappa and mu opioid receptors; however, in humans, naloxone, an opioid antagonist, does not antagonize the analgesic effects of ketamine. Safety and efficacy of ketamine as an anesthetic and analgesic agent is well-documented 2-4. Ketamine is not labeled by the FDA as an analgesic agent. Low (subanesthestic) doses of ketamine have minimal adverse impact upon cardiovascular or respiratory function but produce analgesia and modulate central sensitization, hyperalgesia, and opioid tolerance. Cancer pain, especially in end stages, can be very complicated and is mediated by a variety of pathways: visceral, nociceptive, neuropathic and central. If ketamine can be utilized for pain in end stage cancer patients, this could provide them with superior analgesia and better quality of life, without the risk of significant respiratory depression associated with opioid medications. One of the challenges that we face with ketamine is the route of administration. The most common route is intravascular or intramuscular. Although it has been given orally and rectally, the bioavailability of ketamine when given via these routes is limited to $20-30 \%$. Intranasal (NAS) administration has advantages of being needle free method of administration with potential for outpatient therapy. It lacks hepatic first pass effect resulting in higher bioavailability compared to oral route. Large surface area, uniform temperature, high permeability and extensive vascularity of the nasal mucosa facilitate rapid systemic absorption of intranasal administered drugs 6 . In the pilot trial conducted by the study investigators, single dosage of intranasal ketamine has been shown to be feasibility and effective option for temporary pain reduction in patients with cancer related pain. The investigators now seek to obtain feasibility and efficacy data on long-term use of intranasal ketamine for cancer related pain. Ketamine is a scheduled III medication. A physician with a DEA license can order intranasal ketamine from a compounding pharmacy. Primary outcome of interest: Pain scores will be recorded on Numerical Pain Rating Scale (NPRS) at regular intervals throughout the study. NPRS is the most responsive tool to document pain intensity when compared to Visual Analogue Scale (VAS) and Visual Rating Scale (VRS) for measuring pain, 7 showing higher compliance rates, better responsiveness, ease of use, and good applicability relative to VAS/VRS8. Minimal clinically important differences (MCIDs) for pain ratings varies substantially based on patient population and statistical technique used, range of 0.4 to 3.7 points has been reported as a MCID. In general, improvements of pain severity $</=1.5$ points on NPRS could be seen as clinically irrelevant 9-13. Above that value, the cutoff point for "clinical relevance" depends on patients' baseline pain severity, and ranges from 
2.4 to $5.311-13$. Higher baseline scores require larger raw changes to represent clinically important differences 14 . Primary aim: To determine efficacy of intranasal ketamine in reducing cancer related pain. A clinical trial will be conducted to determine effect of intranasal ketamine on cancer related pain. Pain scores will be recorded on Numerical Pain Rating Scale (NPRS) at regular intervals throughout the study. Minimal clinically important differences (MCIDs) for pain ratings varies substantially based on patient population and statistical technique used, range of 0.4 to 3.7 points has been reported as a MCID. In general, improvements of pain severity $</=1.5$ points on NPRS could be seen as clinically irrelevant 9-13. Above that value, the cutoff point for "clinical relevance" depends on patients' baseline pain severity, and ranges from 2.4 to 5.3 11-13. Higher baseline scores require larger raw changes to represent clinically important differences 14 . Several clinical trials for pain have reported a reduction of 2 points on NPRS to be clinically important.15-17 Therefore for the purposes of this study, MCID of 2 was used for sample size calculations. A prior research study done by Carr et al. studied effects of intranasal ketamine for breakthrough pain in patients with chronic pain of various etiologies. 18 Total number of subjects in this study was 20 (4 of these had cancer related pain).This study demonstrated a mean reduction of 2.7 units on NPRS $(\mathrm{P}<0.0001)$, with standard deviation of 1.87 . Since MCID is 2, effect size using this $(\mathrm{MCID} / \mathrm{SD})=1.05$. Power and sample size table: Assumptions: 1. T-test is the appropriate test (may not be the appropriate test since we have a small sample size and may not be able to assume normality of means based on the central limit theorem) 2. Distribution of reductions in pain score is normal 3. Effect size of 1.05 is clinically meaningful; Sample Size: A sample size of 7 from a population of 20 (in the study done by Carr et al.) achieves $80 \%$ power to detect a NPRS difference of -2 between the null hypothesis mean of 0.0 and the alternative hypothesis mean of 2 with an estimated standard deviation (SD) of 1.87 and with a significance level (alpha) of 0.05 using paired t-test assuming that the actual distribution is normal. We will include 10 patients to account for the possibility that the observed pain reduction in the current study may be different than the study done by Carr, as in this study patients were given ketamine for breakthrough pain, as opposed to for baseline pain. We will enroll 25 patients in the study to account for potential dropouts. RESULTS/ANTICIPATED RESULTS: Majority of subjects experienced the largest decrease in their pain with the 10mg IV dose. Side effects included nausea/vomiting and a feeling of unreality. All side effects resolved by the end of each study visit. No severe adverse events occurred. DISCUSSION/SIGNIFICANCE OF IMPACT: Further study is required to elucidate safety of NAS ketamine with long term use for cancer related pain.

3178

Effects of Motor Skill Training vs. Strength and Flexibility Exercise on Functional Limitations, Pain, and Movement Characteristics in People with Chronic Low Back Pain

Quenten L Hooker ${ }^{1}$, Kristen Roles ${ }^{1}$, Vanessa M. Lanier ${ }^{1}$ and Linda R. Van Dillen ${ }^{1}$

${ }^{1}$ Washington University in St. Louis

OBJECTIVES/SPECIFIC AIMS: Compare the short- and long-term effects of 2 treatments, MST and SF, on limitations in function, pain, and movement characteristics. The movement characteristics included the amount of early excursion ( $1^{\text {st }}$ half of decent) of the knee, hip, and lumbar spine during a functional activity test of picking up an object. METHODS/STUDY POPULATION: 154 participants were randomized to 6, 1-hour treatment sessions (once/ week for 6 weeks) of MST or SF. The MST group received individualized training to modify pain-provoking altered movement patterns during functional activities. The SF group received exercises for trunk strength and trunk and limb flexibility. At baseline, post-treatment and 6-month follow-up participants completed the modified Oswestry Disability Questionnaire (MODQ, a functional limitation measure; 0-100\%), the Numeric Pain Rating Scale (NRS, average pain prior 7 days; $0-10$ ) and a standardized pick up an object test, where sagittal plane knee, hip and lumbar spine excursion were calculated using $3 \mathrm{D}$ motion capture. A mixed model repeated measures ANOVA was used to examine the following effects: Treatment group (Tx), Time and Tx X Time for each self-report and movement variable. When the ANOVA was significant $(\mathrm{p}<0.05)$, a priori planned contrasts were examined. RESULTS/ANTICIPATED RESULTS: There was a significant Tx X Time interaction $(\mathrm{p}<0.01)$ for each outcome. Baseline: MST and SF were similar in MODQ scores $[\Delta 0.4 \%(-3.4-2.9)]$, NRS $[\Delta 0.0(-0.6-0.6)]$, knee $\left[\Delta 2.2^{\circ}\right.$ $(-6.7-2.5)]$, hip $\left[\Delta 0.4^{\circ}(-2.9-2.5)\right]$, and lumbar spine $\left[\Delta 0.1^{\circ}\right.$ $(-1.4-1.2)]$ early excursion. Post-Treatment: Both group's MODQ and NRS scores decreased ( $\mathrm{p}<0.01)$, but MST had a greater reduction in MODQ scores $[\Delta-7.6 \%(-3.9--11.0)]$ and lower average NRS scores $[\Delta-0.8(-0.1--1.4)]$ compared to SF. MST changed knee $\left[\Delta+18.6^{\circ}(14.6-22.1)\right]$, hip $\left[\Delta+10.8^{\circ}(8.5-13.1)\right]$, and lumbar spine $\left[\Delta-2.0^{\circ}(-3.0--1.0)\right]$ early excursion, while SF did not change early joint excursion (all $\mathrm{p}>0.72$ ). 6-Month Follow-up: The differences between MST and SF were maintained for all outcomes $(\mathrm{p}>0.26)$. DISCUSSION/SIGNIFICANCE OF IMPACT: MST was more effective at reducing functional limitations and pain and improving movement patterns during a functional activity compared to SF. For all variables, the differences identified during treatment between MST and SF were maintained at 6-month follow-up. Therefore compared to SF, MST that targets performance of altered movement patterns during functional activities appears to be superior for attaining and maintaining changes in functional limitations, pain, and movement characteristics in people with CLBP.

3010

\section{Effects of non-invasive brain stimulation on speech fluency and brain activity in adults who stutter: a randomized controlled clinical trial}

Emily O'Dell Garnett, Ph.D., CCC-SLP ${ }^{1}$, Soo-Eun Changv, Benjamin Hampstead ${ }^{1}$ and Ho Ming Chow ${ }^{1}$

${ }^{1}$ University of Michigan School of Medicine

OBJECTIVES/SPECIFIC AIMS: The goal of this study is to measure speech fluency and brain activity before and after 5 days of behavioral speech fluency training alone (sham group) or speech training plus stimulation (active group). A 1-month follow up will also be completed. The first primary outcome measure is changes in brain activation in speech motor control/timing network. The second primary outcome measure is changes in percentage of stuttered syllables during speech sample (speech fluency). The secondary outcome measure is changes from baseline on the Overall Assessment of Speakers Experience of Stuttering (OASES), a detailed subject rating of how stuttering affects their lives. METHODS/STUDY POPULATION: This study is a between subjects, counterbalanced, sham-controlled, double-blind design. Participants will be 40 adults who stutter who will be randomized (using minimization) into either the active or 